\title{
Image guided high-dose-rate brachytherapy versus volumetric modulated arc therapy for head and neck cancer: A comparative analysis of dosimetry for target volume and organs at risk
}

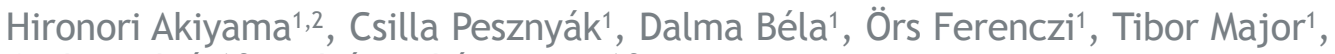 \\ Csaba Polgár ${ }^{1,3}$, Zoltán Takácsi-Nagy ${ }^{1,3}$
}

\author{
${ }^{1}$ Center of Radiotherapy, National Institute of Oncology, Budapest, Hungary \\ ${ }^{2}$ Department of Oral Radiology, Osaka Dental University, Osaka, Japan \\ ${ }^{3}$ Department of Oncology, Semmelweis University, Budapest, Hungary
}

Radiol Oncol 2018; 52(4): 461-467.

Received 19 June 2018

Accepted 6 October 2018

Correspondence to: Hironori Akiyama, Department of Oral Radiology, Osaka Dental University, 1-5-17 Otemae Chuo-Ku, 5400008, Osaka, Japan. Phone: +81-90-5894-3937; E-mail: ddiisbt@gmail.com

Disclosure: No potential conflicts of interest were disclosed.

Background. The aim of the study was to present dosimetric comparison of image guided high-dose-rate brachytherapy (IGBT) with volumetric modulated arc therapy (VMAT) for head and neck cancer regarding conformity of dose distribution to planning target volume (PTV) and doses to organs at risk (OARs).

Patients and methods. Thirty-eight consecutive patients with T1-4 mobile tongue, floor of mouth and base of tongue cancer treated with IGBT were selected. For these patients additional VMAT treatment plans were also prepared using identical computed tomography data. OARs and PTV related parameters (e.g. V98, D0.1 $\mathrm{cm}^{3}$, Dmean, etc.) were compared.

Results. Mean V98 of the PTV was $90.2 \%$ vs. $90.4 \%$ ( $p>0.05)$ for IGBT and VMAT, respectively. Mean D0.1 $\mathrm{cm}^{3}$ to the mandible was $77.0 \%$ vs. $85.4 \%$ ( $p<0.05$ ). Dmean to ipsilateral and contralateral parotid glands was $4.6 \%$ vs. $4.6 \%$ and $3.0 \%$ vs. 3.9\% ( $p>0.05$ ). Dmean to ipsilateral and contralateral submandibular glands was $16.4 \%$ vs. $21.9 \%(p>0.05)$ and $8.2 \%$ vs. $16.9 \%(p<0.05)$, respectively.

Conclusions. Both techniques showed excellent target coverage. With IGBT dose to normal tissues was lower than with VMAT. The results prove the superiority of IGBT in the protection of OARs and the important role of this invasive method in the era of new external beam techniques.

Key words: head and neck cancers; image guided high-dose-rate brachytherapy; volumetric modulated arc therapy; dosimetric comparison

\section{Introduction}

Head and neck $(\mathrm{H} \& \mathrm{~N})$ cancer is an excellent indication for radiation therapy (RT) which is an organ preserving method maintaining the quality of life of patients. Brachytherapy (BT) with or without external beam RT (EBRT) can play an essential role in the treatment of certain tumor localizations in the H\&N area except cases with bone invasion or proximity of large vessels, delivering an ablative dose to the target volume while sparing the critical and normal tissues - due to the rapid dose fall-off - which is not safely feasible with EBRT alone. ${ }^{1-3}$ Image guided high-dose-rate BT (IGBT) with computed tomography $(\mathrm{CT})$ and/or magnetic resonance imaging (MRI) has been implemented improving the effica- 
cy of BT. ${ }^{4}$ IGBT decreases irradiated doses to critical structures without compromising target coverage. ${ }^{5}$ However, IGBT is an invasive procedure requiring special skills and interdisciplinary co-operation with a H\&N surgeon, as well as patience on the part of patients who have to endure e the insertion of catheters. ${ }^{6,7}$ Recently, intensity modulated RT (IMRT) has been introduced in clinical practice and achieved higher dose conformity with better organs at risk (OARs) sparing compared to 3-dimensional conformal radiation therapy. ${ }^{8}$ Volumetric modulated arc therapy (VMAT), an improved technique of IMRT offers reduced irradiation time compared to IMRT. ${ }^{9}$ Nowadays IGBT meets the challenge of high precision EBRT such as VMAT, which is a non-invasive modality and does not require special skills to deliver a high conformal dose to the target volume while saving critical normal structures. To our knowledge no detailed dosimetric comparisons of IGBT with VMAT have so far been reported in the H\&N region, such publications are available only for breast and cervical cancer. ${ }^{10,11}$

The purpose of this study is to present a dosimetric analysis regarding planning target volume (PTV) and OARs with the comparison of IGBT and VMAT for localized H\&N cancer using identical CT data and contours.

\section{Patients and methods}

\section{Patients' characteristics}

Thirty-eight consecutive patients with T1-4 mobile tongue $(n=17)$, floor of mouth $(n=9)$ and base of tongue $(\mathrm{n}=12)$ cancer treated between January 2013 and March 2017 at the National Institute of Oncology, Budapest, Hungary were selected for this study (Table 1). Primary lesions or tumor bed were treated with CT image based IGBT alone (n $=22)$ or after EBRT as a boost $(\mathrm{n}=16)$. All T3-4

TABLE 1. Dosimetry of PTV

\begin{tabular}{|c|c|c|c|c|c|}
\hline \multirow{2}{*}{ Parameters } & \multicolumn{2}{|c|}{$\begin{array}{l}\text { IGBT } \\
\mathrm{n}=38\end{array}$} & \multicolumn{2}{|c|}{$\begin{array}{l}\text { VMAT } \\
n=38\end{array}$} & \multirow{2}{*}{$p$-value } \\
\hline & mean & S.D. & mean & S.D. & \\
\hline V95 (\%) & 92.1 & 3.0 & 98.4 & 0.9 & $<0.05$ \\
\hline V98 (\%) & 90.2 & 3.2 & 90.4 & 3.7 & $>0.05$ \\
\hline V100 (\%) & 89.0 & 3.4 & 76.7 & 8.9 & $<0.05$ \\
\hline D90 (\%) & 98.6 & 4.7 & 98.2 & 0.8 & $<0.05$ \\
\hline D100 (\%) & 58.6 & 9.0 & 87.0 & 3.2 & $<0.05$ \\
\hline
\end{tabular}

$\mathrm{DX}=$ minimum relative dose of the planning dose delivered to $\mathrm{X} \%$ of the PTV; IGBT = image guided high-dose-rate brachytherapy; $n=$ number of patients for analysis; PTV = planning target volume; S.D. = standard deviation; $\mathrm{VMAT}=$ volumetric modulated arc therapy; $\mathrm{VX}=$ relative volume of the PTV receiving at least $\mathrm{X} \%$ of the panning dose cases $(n=12)$ were base of tongue cancer treated exclusively with radiotherapy (EBRT + BT boost). The T4 tumors invaded the deep muscles of the tongue, without invasion into the mandible or other regions. Tumor excision was carried out in 18 patients. Ipsi- and contralateral submandibular glands (15 and 1) (iSMGs, cSMGs) were removed during surgery.

\section{Brachytherapy planning}

The process of BT planning was described in details in our previous publication. ${ }^{12}$ Under general anesthesia in the operating theatre plastic catheters (median 7, range 3-12) were implanted into the target volume located in 17 patients on the left, in 18 on the right side, and in 3 in the central region of the tongue/ floor of mouth/base of tongue. After implantation, all patients underwent CT imaging with $3 \mathrm{~mm}$ slice thickness including the primary tumor or tumor bed, the spinal cord, parotid glands (PAGs) on both sides and SMGs. The images were transferred to the Oncentra Brachy v4.3 (Elekta, Brachytherapy, Veenendaal, The Netherlands) treatment planning system. Gross tumor volume (GTV), clinical target volume (CTV) and PTV were contoured on CT images as follows. In non-surgical cases (definitive) palpation, visual inspection and MR images without CT-MRI fusion were used to determine GTV in the patients. CTV was defined as GTV $+5 \mathrm{~mm}$ limited to mandible. In postoperative cases CTV was directly defined similarly to definitive cases using the preoperative MRI. For all cases PTV was the same as CTV without using any margin. Based on CT image sets, the mandible, the spinal cord, PAGs on both sides and SMGs - as OARs - were delineated. Mandible was not part of the PTV. After catheter reconstruction, treatment plans were made with geometrical optimization, complemented with graphical optimization by adjusting the isodose line in order to appropriately cover the PTV by the prescribed dose (PD) and keep the doses to OARs as low as possible. ${ }^{13}$ Our aim was to obtain less than 0.40 for the dose non-uniformity ratio (DNR), the ratio of volumes receiving 1.5 times the $\mathrm{PD}$ and those receiving the PD, $\left(\mathrm{V}_{150} / \mathrm{V}_{100}\right)$. At our institute the fractionation schedule was 15 x 3 Gy (45 Gy) for IGBT alone and 7 x 3 Gy (21 Gy) for IGBT after 50 Gy EBRT.

\section{VMAT planning}

For VMAT plans the same CT images and structure set were used as for IGBT. The contours applied for 
IGBT plans were transferred from the Oncentra Brachy to the Eclipse v11 (Varian Medical Systems, Palo Alto, CA) treatment planning system using DICOM RT protocol. Due to this process, all contours of PTV and OARs were identical in both planning systems. In case of IGBT alone patients VMAT was set up for a total dose of 70 Gy (35 x 2 Gy), and for EBRT+IGBT patients a total dose of $20 \mathrm{~Gy}(10 \mathrm{x}$ 2 Gy) was planned as a boost. VMAT plans were created with $6 \mathrm{MV}$ photon energy beams using double partial arcs from gantry angle $110^{\circ}$ to $250^{\circ}$ to ensure the homogeneity of PTV dose coverage. As regards the dose constraints for OARs and target volume coverage, VMAT plans were optimized using the Varian RapidArc progressive resolution optimization algorithm (PRO) and AAA dose calculation algorithm. The objective for PTV was to deliver a dose of $95-107 \%$ of the PD. The dose constraints for PTV70Gy were as follows: V98\% > 90\%, $\mathrm{V} 95 \%>95 \%, \mathrm{~V} 50 \%=100 \%, \mathrm{~V} 2 \%<107 \%$, for the spinal cord V2\% < $45 \mathrm{~Gy}$, for parotid glands Dmean $<24 \mathrm{~Gy}$, for mandible V0.03cc $<70 \mathrm{~Gy}$.

\section{Dosimetric analysis}

Based on dose-volume-histograms (DVHs), treatment plans of both techniques were dosimetrically analyzed. For evaluating conformity of dose distribution to PTV, the relative volume of the PTV receiving at least 95, 98 and $100 \%$ of the PD (V95, V98 and V100) and the minimum relative dose of the PD delivered to 90 and 100\% of the PTV (D90 and D100) were calculated. The minimum relative doses of the PD delivered to the most exposed 0.1, 1 and $2 \mathrm{~cm}^{3}$ of the organs $\left(\mathrm{D} 0.1 \mathrm{~cm}^{3}, \mathrm{D} 1 \mathrm{~cm}^{3}\right.$ and D2 $\mathrm{cm}^{3}$ ) were quantified for the assessment of doses to OARs. The salivary glands were classified as ipsilateral or contralateral based on the tumor location, and were dosimetrically evaluated. For the salivary glands, additional parameters such as the mean relative dose of the PD delivered to the organs (Dmean), D10, D30, D50, V10, V30 and V50 were also quantified. The dosimetric data for the parotid and submandibular glands were not evaluated for all patients, because in some cases the glands were excluded from the field-of-view of the CT scans, the volume was too small (for D1 and D2 $\mathrm{cm}^{3}$ calculation), or the target volume (tumor or tumor bed) was centrally located (Tables 4 and 5). The spinal cord was not detected in one case because it was excluded from the field-of-view of the CT scans (Table 3).

The mean value with standard deviation was used to describe dosimetric parameters for both
TABLE 2. Dosimetry of mandible and spinal cord

\begin{tabular}{|c|c|c|c|c|c|}
\hline \multirow{2}{*}{ Parameters } & \multicolumn{2}{|c|}{ IGBT } & \multicolumn{2}{|c|}{ VMAT } & \multirow{2}{*}{$p$-value } \\
\hline & mean & S.D. & mean & S.D. & \\
\hline \multicolumn{6}{|l|}{ Mandible, $n=38$} \\
\hline $\mathrm{D} 0.1 \mathrm{~cm}^{3}(\%)$ & 77.0 & 17.2 & 85.4 & 7.9 & $<0.05$ \\
\hline $\mathrm{Dlcm} \mathrm{cm}^{3}(\%)$ & 56.9 & 13.4 & 74.5 & 9.6 & $<0.05$ \\
\hline $\mathrm{D} 2 \mathrm{~cm}^{3}(\%)$ & 48.4 & 12.2 & 68.4 & 9.5 & $<0.05$ \\
\hline \multicolumn{6}{|c|}{ Spinal cord, $n=37^{*}$} \\
\hline $\mathrm{D} 0.1 \mathrm{~cm}^{3}(\%)$ & 9.7 & 2.6 & 12.3 & 4.1 & $<0.05$ \\
\hline $\mathrm{Dlcm}^{3}(\%)$ & 6.8 & 2.0 & 10.8 & 3.7 & $<0.05$ \\
\hline $\mathrm{D} 2 \mathrm{~cm}^{3}(\%)$ & 5.9 & 1.9 & 10.0 & 3.6 & $<0.05$ \\
\hline
\end{tabular}

$\mathrm{DXcm}{ }^{3}=$ minimum relative dose of the planning dose delivered to most exposed $\mathrm{X} \mathrm{cm}^{3}$ of the organs; IGBT = image guided high-dose-rate brachytherapy; $n=$ number of patients for analysis; S.D. = standard deviation; VMAT = volumetric modulated arc therapy.

* in one patient the spinal cord was not detected because it was excluded from the field-of-view of the CT scans

TABLE 3. Dosimetry of parotid gland

\begin{tabular}{|c|c|c|c|c|c|}
\hline \multirow{2}{*}{ Parameters } & \multicolumn{2}{|c|}{ IGBT } & \multicolumn{2}{|c|}{ VMAT } & \multirow{2}{*}{$p$-value } \\
\hline & mean & S.D. & mean & S.D. & \\
\hline \multicolumn{6}{|c|}{ ipsilateral side $\left(n=34^{*}\right)$} \\
\hline Dmean (\%) & 4.6 & 1.4 & 4.6 & 2.6 & $>0.05$ \\
\hline $\mathrm{D} 0.1 \mathrm{~cm}^{3}(\%)$ & 11.2 & 2.8 & 18.0 & 7.1 & $<0.05$ \\
\hline $\mathrm{Dlcm}^{3}(\%)$ & 8.1 & 2.1 & 12.9 & 6.3 & $<0.05$ \\
\hline $\mathrm{D} 2 \mathrm{~cm}^{3}(\%)$ & 7.0 & 2.0 & 10.5 & 5.7 & $<0.05$ \\
\hline D10 (\%) & 7.3 & 2.1 & 11.2 & 5.6 & $<0.05$ \\
\hline D30 (\%) & 5.5 & 1.6 & 5.5 & 4.0 & $>0.05$ \\
\hline D50 (\%) & 4.5 & 1.3 & 3.0 & 3.1 & $<0.05$ \\
\hline V10 (\%) & 3.1 & 4.0 & 18.0 & 16.9 & $<0.05$ \\
\hline V30 (\%) & 0.0 & 0.0 & 0.7 & 3.9 & N.A. \\
\hline V50 (\%) & 0.0 & 0.0 & 0.1 & 0.5 & N.A. \\
\hline \multicolumn{6}{|c|}{ contralateral side $\left(n=35^{* *}\right)$} \\
\hline Dmean (\%) & 3.0 & 0.9 & 3.9 & 2.1 & $>0.05$ \\
\hline $\mathrm{D} 0.1 \mathrm{~cm}^{3}(\%)$ & 8.9 & 2.1 & 15.3 & 5.9 & $<0.05$ \\
\hline $\mathrm{Dlcm}^{3}(\%)$ & 5.9 & 1.6 & 10.8 & 5.9 & $<0.05$ \\
\hline $\mathrm{D} 2 \mathrm{~cm}^{3}(\%)$ & 4.9 & 1.5 & 9.1 & 5.4 & $<0.05$ \\
\hline D10 (\%) & 5.4 & 1.3 & 9.9 & 4.4 & $<0.05$ \\
\hline D30 (\%) & 4.0 & 1.0 & 4.9 & 3.7 & $>0.05$ \\
\hline D50 (\%) & 3.2 & 0.8 & 2.2 & 2.3 & $<0.05$ \\
\hline V10 (\%) & 0.5 & 0.3 & 14.2 & 13.2 & $<0.05$ \\
\hline V30 (\%) & 0.0 & 0.0 & 0.8 & 4.7 & N.A. \\
\hline V50 (\%) & 0.0 & 0.0 & 0.2 & 1.3 & N.A. \\
\hline
\end{tabular}

Dmean $=$ minimum relative dose of the planning dose delivered to the organs; $D X=$ minimum relative dose of the planning dose delivered to $\mathrm{X} \%$ of the PTV; $\mathrm{DXcm}^{3}=$ minimum relative dose of the planning dose delivered to most exposed $X \mathrm{~cm}^{3}$ of the organs; IGBT = image guided highdose-rate brachytherapy; $n=$ number of patients for analysis; N.A. = not available; S.D. = standard deviation; $V M A T$ = volumetric modulated arc therapy; $V X=$ relative volume of the PTV receiving at least $\mathrm{X} \%$ of the panning dose

" in one patient the parotid gland was not detected because it was excluded from the fieldof-view of the CT scans, and in three patients parotid glands were not evaluated because of central implantation

"*three patients were not evaluated because of central implantation 

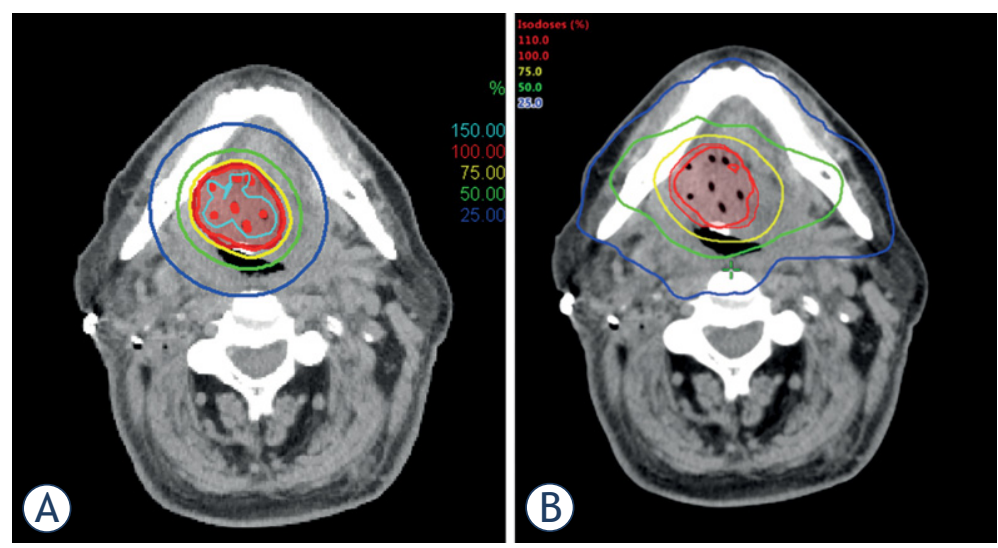

FIGURE 1. Representative dose distribution of (A) image guided high-dose-rate brachytherapy (IGBT) and (B) volumetric modulated arc therapy (VMAT).

TABLE 4. Dosimetry of submandibular gland

\begin{tabular}{|c|c|c|c|c|c|}
\hline \multirow{2}{*}{ Parameters } & \multicolumn{2}{|c|}{ IGBT } & \multicolumn{2}{|c|}{ VMAT } & \multirow{2}{*}{$p$-value } \\
\hline & mean & S.D. & mean & S.D. & \\
\hline \multicolumn{6}{|l|}{ ipsilateral side } \\
\hline Dmean $(\%), n=20^{*}$ & 16.4 & 10.7 & 21.9 & 19.9 & $>0.05$ \\
\hline $\mathrm{D} 0.1 \mathrm{~cm}^{3}(\%), \mathrm{n}=20^{*}$ & 27.4 & 15.6 & 39.9 & 23.3 & $>0.05$ \\
\hline $\mathrm{Dlcm}{ }^{3}(\%), \mathrm{n}=18^{* *}$ & 19.4 & 11.2 & 27.2 & 17.6 & $>0.05$ \\
\hline $\mathrm{D} 2 \mathrm{~cm}^{3}(\%), \mathrm{n}=17^{* *}$ & 16.1 & 9.4 & 20.8 & 14.7 & $>0.05$ \\
\hline $\mathrm{D} 10(\%), \mathrm{n}=20^{*}$ & 22.6 & 13.9 & 33.2 & 23.0 & $>0.05$ \\
\hline D30 (\%), $n=20^{*}$ & 18.3 & 11.7 & 25.8 & 21.9 & $>0.05$ \\
\hline $\mathrm{D} 50(\%), \mathrm{n}=20^{*}$ & 15.7 & 10.4 & 21.0 & 20.9 & $>0.05$ \\
\hline V10 (\%), n=20* & 61.3 & 38.6 & 63.9 & 33.6 & $>0.05$ \\
\hline $\mathrm{V} 30(\%), \mathrm{n}=20^{*}$ & 14.5 & 28.0 & 30.1 & 38.7 & $>0.05$ \\
\hline V50 (\%), $n=20^{*}$ & 1.4 & 3.7 & 14.1 & 27.4 & $>0.05$ \\
\hline \multicolumn{6}{|l|}{ contralateral side } \\
\hline Dmean (\%), $n=34^{* * *}$ & 8.2 & 4.8 & 16.9 & 10.8 & $<0.05$ \\
\hline $\mathrm{D} 0.1 \mathrm{~cm}^{3}(\%), \mathrm{n}=34^{* * *}$ & 13.4 & 5.3 & 29.7 & 12.0 & $<0.05$ \\
\hline $\mathrm{Dlcm}^{3}(\%), \mathrm{n}=32^{* * * *}$ & 9.4 & 3.2 & 21.7 & 8.6 & $<0.05$ \\
\hline $\mathrm{D} 2 \mathrm{~cm}^{3}(\%), \mathrm{n}=32^{* * * *}$ & 8.1 & 2.9 & 18.3 & 8.3 & $<0.05$ \\
\hline $\mathrm{D} 10(\%), \mathrm{n}=34^{* * *}$ & 11.1 & 5.6 & 25.3 & 12.2 & $<0.05$ \\
\hline $\mathrm{D} 30(\%), \mathrm{n}=34^{* * *}$ & 9.2 & 5.1 & 20.4 & 11.4 & $<0.05$ \\
\hline $\mathrm{D} 50(\%), \mathrm{n}=34^{* * *}$ & 8.0 & 4.7 & 16.7 & 11.4 & $<0.05$ \\
\hline $\mathrm{V} 10(\%), \mathrm{n}=34^{* * *}$ & 26.0 & 30.2 & 69.9 & 27.5 & $<0.05$ \\
\hline V30 (\%), $n=34^{* * *}$ & 1.2 & 7.0 & 14.1 & 25.1 & $<0.05$ \\
\hline V50 (\%), $\mathrm{n}=34^{* * *}$ & 0.0 & 0.0 & 2.7 & 12.1 & N.A. \\
\hline
\end{tabular}

Dmean $=$ minimum relative dose of the planning dose delivered to the organs; $\mathrm{DX}=$ minimum relative dose of the planning dose delivered to $\mathrm{X} \%$ of the PTV; $\mathrm{DXcm}^{3}=$ minimum relative dose of the planning dose delivered to most exposed $X \mathrm{~cm}^{3}$ of the organs; IGBT = image guided highdose-rate brachytherapy; $n=$ number of patients for analysis; N.A. = not available; S.D. = standard deviation; $\mathrm{VMAT}=$ volumetric modulated arc therapy; $\mathrm{VX}=$ relative volume of the PTV receiving at least $\mathrm{X} \%$ of the panning dose

in fifteen patients the submandibular glands were resected by operation and three patients were not evaluated because of central implantation.

.. two and three patients, respectively, were additionally excluded from the analysis of $\mathrm{Dl} \mathrm{cm}^{3}$ and D2 $\mathrm{cm}^{3}$ because the volume was too small.

$\cdots$ in one patient the submandibular gland was resected by operation and three patients were not evaluated because of central implantation.

.... two and two patients, respectively, were additionally excluded from the analysis of $\mathrm{Dlcm} \mathrm{cm}^{3}$ and D2 $\mathrm{cm}^{3}$ because the volume was too small. techniques, and the data were compared using the non-parametric Mann-Whitney $U$ test with GraphPad Prism version 5.01 for Windows (GraphPad Software, San Diego, CA). The level of statistical significance was set at $p \leq 0.05$.

\section{Results}

The dosimetric parameters of PTV are presented in Table 2. Dose distribution of BT and VMAT are presented in Figure 1. The dose conformity was excellent with both techniques. V95 was significantly better with VMAT (98.4\% vs. $92.1 \%, p<0.05)$, but V100 was superior with IGBT $(89.0 \%$ vs. $76.7 \%, p<$ 0.05). V98 was similar in both techniques $(90.2 \%$ vs. $90.4 \%$ for IGBT and VMAT, $\mathrm{p}>0.05$, respectively). Relative dose coverage to PTV, D90 was statistically significantly better with IGBT (98.6\% vs. $98.2 \%)$, probably without clinical consequences. However, D100 showed a reverse result (58.6\% vs. $87.0 \%)$.

As for OARs, the mandible and the spinal cord were better protected by IGBT (Table 3). For doses to small volumes (D0.1 $\mathrm{cm}^{3}, \mathrm{D} 1 \mathrm{~cm}^{3}$ and $\left.\mathrm{D} 2 \mathrm{~cm}^{3}\right)$ significantly lower values were obtained with IGBT (e.g. D0.1 $\mathrm{cm}^{3}$ was $77.0 \%$ vs. $85.4 \%$ and $9.7 \%$ vs. $12.3 \%$ to the mandible and the spinal cord).

Doses to the salivary glands were generally lower with IGBT (Tables 4 and 5). Six parameters of ipsilateral PAGs (iPAGs) were significantly lower with IGBT than with VMAT $\left(e . g\right.$. D0.1 $\mathrm{cm}^{3}$ was $11.2 \%$ vs. $18.0 \%$, D10 was $7.3 \%$ vs. $11.2 \%$ and $\mathrm{V} 10$ was $3.1 \%$ vs. $18.0 \%$, respectively) (Table 4 ). Two parameters (Dmean and D30) were identical for the two techniques (4.6\% and 5.5\%). Parameters of contralateral PAGs (cPAGs) showed the same tendency as iPAGs (Table 4). Dosimetry of iSMGs indicated that doses with IGBT were smaller than with VMAT, but no significant differences were found between the two techniques (Table 5). All dose parameters of cSMGs were significantly smaller with IGBT than with VMAT (e.g. Dmean was $8.2 \%$ vs. $16.9 \%$, D10 was $11.1 \%$ vs. $25.3 \%$ and $\mathrm{V} 10$ was $26.0 \%$ vs. $69.9 \%$, respectively) (Table 5).

\section{Discussion}

The comparison of new technologies in the H\&N region is a very interesting area of research. ${ }^{7}$ Sresty et al. compared plans of IGBT and IMRT for mobile tongue cancer directly, and found a very good dose conformity between the two techniques. ${ }^{14}$ Our study also revealed good target coverage with both 
IGBT and VMAT. Our results confirmed that the volumes irradiated by $100 \%$ of the PD were larger and the dose gradient around PTV was higher with IGBT than with VMAT - because of the physical characteristics of BT. Moreover, IGBT yielded better values in D90, but worse in D100. The majority of locoregional treatment failures occur within the target volumes irradiated with high doses by IMRT. ${ }^{15,16}$ These regions are thought to represent areas of hypoxia and radiation resistant tumor cells, and may require higher doses to improve local control. ${ }^{17}$ These observations might support the theory that the PTV of the primary tumor or tumor bed - if it is technically feasible - can be treated with IGBT.

Owosho et al. analyzed oral and oropharyngeal cancer patients and $96 \%$ of the osteoradionecrosis (ORN) affected regions of the jaw received doses over $60 \mathrm{~Gy}$, suggesting >60 Gy as a threshold for ORN risk. ${ }^{18}$ In the current research mean $D 0.1 \mathrm{~cm}^{3}$ of the mandible was $85.4 \%$ with VMAT, so $0.1 \mathrm{~cm}^{3}$ received at least $59.8 \mathrm{~Gy}$ (70 Gy x 0.854). The mandible was significantly better protected with IGBT than with VMAT, due to intensity modulation with stepping source technology avoiding high irradiation of the mandible. ${ }^{1}$ IGBT provided safe and secure treatment for the mandible.

For tonsillar cancer patients Stieler et al. reported 41.6 Gy and 42.6 Gy as mean maximum dose to the spinal cord with IMRT (9 fields) and VMAT (single arc), respectively, including the neck region in the treatment fields..$^{19}$ In the direct comparison of plans of IGBT and IMRT for mobile tongue cancer, Sresty et al. reported maximum doses to the spinal cord from $9 \%$ to $14 \%$ with IGBT, whereas from $15.6 \%$ to $24.6 \%$ with IMRT. ${ }^{14}$ Meanwhile our results of mean $\mathrm{D} 0.1 \mathrm{~cm}^{3}$, which represents the maximum dose to the spinal cord, showed $9.7 \%$ (4.4 Gy) with IGBT and $12.3 \%$ (8.6 Gy) with VMAT $(p<0.05)$. The results were similar and doses to the spinal cord were acceptable for both techniques in patients, in whom only the primary lesion was irradiated.

Dose to PAGs is important in terms of xerostomia, because they produce up to $70 \%$ of the total stimulated saliva. ${ }^{20-23}$ Owosho et al. analyzed the role of PAGs irradiation in the development of severe xerostomia defined as Grade 4 according to the LENT SOMA scales after IMRT, and reported that xerostomia occurred in a follow-up time of $<6$ months, when the Dmean to iPAG and cPAG was 43.8 Gy and 24.9 Gy, respectively..$^{23}$ They concluded that the incidence of xerostomia could be decreased by limiting the mean dose to both PAGs to values below $25 \mathrm{~Gy}$. In our study the mean doses to iPAG and cPAG were 4.6\% (3.2 Gy) and 3.9\% (2.7 Gy) with VMAT and 4.6\% (2.1 Gy) and 3.0\% (1.4 Gy) with IGBT, respectively. Dose delivery to PAGs by both techniques probably has a little impact on xerostomia in patients, whose PTV includes only the primary tumor or tumor bed, but in IGBT the dose to PAGs was somewhat lower. Maintenance of adequate SMG function is also important in the reduction of xerostomia because SMGs produce about $20 \%$ to $30 \%$ of salivary output, including up to $90 \%$ of unstimulated salivary output. ${ }^{24-27}$ Wang et al. reported SMG dosimetry using IMRT with or without cSMG sparing. ${ }^{27}$ In their study the mean doses to iSMGs and cSMGs were 60.8 Gy and 20.4 Gy, in the cSMG-sparing group as opposed to the unspared group, where these parameters were 60.9 Gy and 57.4 Gy, respectively. They observed that xerostomia grades at 2 and 6 months post-IMRT were significantly lower among patients in the cSMG-sparing group than in the unspared group. In our study all parameters of cSMGs were smaller with IGBT than with VMAT $(p<0.05)$. Dmean of cSMGs with VMAT was 16.9\% (11.8 Gy). Dmean of cSMGs with IGBT was 8.2\% (3.7 Gy), about one third of the value with VMAT, so IGBT had a more significant effect on dose reduction to cSMGs.

A major source of concern with VMAT and IMRT is the higher low dose radiation to surrounding normal tissue, which potentially increases the risk of secondary malignancy. ${ }^{17}$ As for our results, V10 with VMAT was appreciably large especially for PAG. Immobilization for precise irradiation and the possibility of tumor repopulation during the long treatment time can also be a problem with VMAT. On the other hand, technique sensitivity is one of the drawbacks of IGBT. However, some measures have been introduced for easy implantation, such as the use of a vinyl template or ultrasound guided technique. ${ }^{5}$

One of the limitations of our study is that the Task Group (TG)-43 formalism, implemented in our planning system for dose calculation, has not taken into consideration tissue heterogeneities. ${ }^{28}$ However, Peppa et al. revealed that the absolute differences in the parameters are too small to warrant clinical importance in terms of tumor control or complication probabilities. ${ }^{29}$ The second drawback is that the catheters in the target have a small tissue inhomogeneity effect. However, according to our investigation the variations in density in small volumes cause less than a $0.5 \%$ change in dosimetry. We are aware of that in EBRT the PTV should be larger than in BT, but our aim was to make comparison between two largely different irradiation 
techniques, and since the same PTVs were used in plans of both treatment modalities in our study the dosimetric results apply only to the differences between BT and VMAT.

Though this article deals with the dosimetric and not the therapeutic comparison of the above mentioned two techniques, we would like to emphasize that the fractionation schedule, which we used in our BT treatment, was based on the European and American recommendations, where the suggested boost dose is $21-30 / 3$ Gy with HDR after $45-50$ Gy EBRT and the definitive dose is 45-60/3 Gy. 2,30 Using alfa/beta $=10 \mathrm{~Gy}$ and without taking into consideration the time factor, the calculated biological effective dose (BED) for BT alone (15 x 3 Gy) was $58.5 \mathrm{~Gy}$, for EBRT ( $25 \times 2 \mathrm{~Gy})$ and BT boost ( 7 x 3 Gy) 87.3 Gy (60 Gy + 27.3 Gy), for EBRT alone (35 x 2 Gy) 84 Gy and for EBRT ( 25 x 2 Gy) and EBRT boost (10 x 2 Gy) 84 Gy (60 Gy + 24 Gy). We applied lower BT dose for BT alone because of the lack of long-term experiences with HDR BT.

\section{Conclusions}

This is the first study with direct dosimetric comparison between IGBT and VMAT for H\&N cancer applying various parameters. Both techniques provided excellent target coverage, but IGBT was found superior in protecting OARs. Adverse events, such as xerostomia and osteoradionecrosis, derived from irradiation of OARs could be serious problems in the $\mathrm{H} \& \mathrm{~N}$ cancer radiotherapy. Therefore, it is clinically important to keep the dose for OARs as low as possible. In this respect the results confirm the important role of interstitial RT in the era of new external beam RT techniques. To translate the results of these dosimetric findings into clinical practice, more patients and long term follow-up with prospective collection of toxicities are necessary.

\section{References}

1. Kovács G. Modern head and neck brachytherapy: from radium towards intensity modulated interventional brachytherapy. J Contemp Brachytherapy 2014; 6: 404-16. doi: 10.5114/jcb.2014.47813

2. Mazeron JJ, Ardiet JM, Haie-Méder C, Kovács G, Levendag P, Peiffert D, et al. GEC-ESTRO recommendations for brachytherapy for head and neck squamous cell carcinomas. Radiother Oncol 2009; 91: 150-6. doi: 10.1016/j. radonc.2009.01.005

3. Takácsi-Nagy Z, Oberna F, Koltai P, Hitre, E, Major T, Fodor J, et al. Longterm outcomes with high-dose-rate brachytherapy for the management of base of tongue cancer. Brachytherapy 2013; 12: 535-41. doi: 10.1016/j. brachy.2013.07.001
4. Major T, Polgár C, Mangel L, Takácsi-Nagy Z, Somogyi A, Németh G. CT based conformal brachytherapy treatment planning. Magy Onkol 2000; 44: 10915. doi: HUON.2000.44.2.0109

5. Yoshida K, Takenaka T, Akiyama H, Yamazaki H, Yoshida M, Masui K, et al. Three-dimensional image-based high-dose-rate interstitial brachytherapy for mobile tongue cancer. J Radiat Res 2014; 55: 154-61. doi: 10.1093/jrr/ rrt079

6. Akiyama H, Yoshida K, Yamazaki H, Takenaka T, Kotsuma, T, Masui, K, et al. High-dose-rate interstitial brachytherapy for mobile tongue cancer: preliminary results of a dose reduction trial. J Contemp Brachytherapy 2014; 6: 10-4. doi: $10.5114 / j c b .2014 .40726$

7. Takácsi-Nagy Z, Martínez-Mongue R, Mazeron JJ, Anker CJ, Harrison LB. American Brachytherapy Society Task Group Report: Combined external beam irradiation and interstitial brachytherapy for base of tongue tumors and other head and neck sites in the era of new technologies. Brachytherapy 2017; 16: 44-58. doi: 10.1016/j.brachy.2016.07.005

8. Gupta T, Agarwal J, Jain S, Phurailatpam R, Kannan S, Ghosh-Laskar S, et al. Three-dimensional conformal radiotherapy (3D-CRT) versus intensity modulated radiation therapy (IMRT) in squamous cell carcinoma of the head and neck: a randomized controlled trial. Radiother Oncol 2012; 104: 343-8. doi: 10.1016/j.radonc.2012.07.001

9. Osborn J. Is VMAT beneficial for patients undergoing radiotherapy to the head and neck? Radiography 2017; 23: 73-6. doi: 10.1016/j. radi.2016.08.008

10. Major T, Stelczer G, Pesznyák C, Mészáros N, Polgár C. Multicatheter interstitial brachytherapy versus intensity modulated external beam therapy for accelerated partial breast irradiation: A comparative treatment planning study with respect to dosimetry of organs at risk. Radiother Oncol 2017; 122: 17-23. doi: 10.1016/j.radonc.2016.08.003

11. Sharma DN, Gandhi AK, Sharma S, Rath GK, Jagadesan P, Julka PK. Interstitial brachytherapy vs. intensity-modulated radiation therapy for patients with cervical carcinoma not suitable for intracavitary radiation therapy. Brachytherapy 2013; 12: 311-6. doi: 10.1016/j.brachy.2012.10.003

12. Akiyama H, Major T, Polgár C, Takácsi-Nagy Z. Dose-volume analysis of target volume and critical structures in computed tomography image-based multicatheter high-dose-rate interstitial brachytherapy for head and neck cancer. J Contemp Brachytherapy 2017; 9: 553-60. doi: 10.5114/jcb.2017.72581

13. Major T, Polgár C. Treatment planning for multicatheter interstitial brachytherapy of breast cancer - from Paris system to anatomy-based inverse planning. J Contemp Brachytherapy 2017; 9: 89-98. doi: 10.5114/jcb.2017.66111

14. Sresty NVNM, Ramanjappa T, Raju AK, Muralidhar KR, Sudarshan G. Acquisition of equal or better planning results with interstitial brachytherapy when compared with intensity-modulated radiotherapy in tongue cancers. Brachytherapy 2010; 9: 235-8. doi: 10.1016/j.brachy.2009.05.006

15. Bussels B, Maes A, Hermans R, Nuyts S, Weltens C, Van den Bogaert $W$. Recurrences after conformal parotid-sparing radiotherapy for head and neck cancer. Radiother Oncol 2004; 72: 119-27. doi: 10.1016/j.radonc.2004.03.014

16. Chao KSC, Ozyigit G, Tran BN, Cengiz M, Dempsey JF, Low DA. Patterns of failure in patients receiving definitive and postoperative IMRT for head-andneck cancer. Int J Radiat Oncol Biol Phys 2003; 55: 312-21.

17. Teoh M, Clark CH, Wood K, Whitaker S, Nisbet A. Volumetric modulated arc therapy: a review of current literature and clinical use in practice. $\mathrm{Br} J$ Radiol 2011; 84: 967-96. doi: 10.1259/bjr/22373346

18. Owosho AA, Tsai CJ, Lee RS, Freymiller $\mathrm{H}$, Kadempour A, Varthis $\mathrm{S}$, et al. The prevalence and risk factors associated with osteoradionecrosis of the jaw in oral and oropharyngeal cancer patients treated with intensitymodulated radiation therapy (IMRT): The Memorial Sloan Kettering Cancer Center experience. Oral Oncol 2017; 64: 44-51. doi: 10.1016/j.oraloncology.2016.11.015

19. Stieler F, Wolff D, Schmid H, Welzel G, Wenz F, Lohr F. A comparison of several modulated radiotherapy techniques for head and neck cancer and dosimetric validation of VMAT. Radiother Oncol 2011; 101: 388-93. doi: 10.1016/j.radonc.2011.08.023

20. Dawes $C$, Wood CM. The contribution of oral minor mucous gland secretions to the volume of whole saliva in man. Arch Oral Biol 1973; 18: 337-42.

21. Sreebny LM. Saliva in health and disease: an appraisal and update. Int Dent J 2000; 50: 140-61. 
22. Humphrey SP, Williamson RT. A review of saliva: normal composition, flow, and function. J Prosthet Dent 2001; 85: 162-9. doi: 10.1067/ mpr.2001.113778

23. Owosho AA, Thor M, Oh JH, Riaz, N, Tsai CJ, Rosenberg H, et al. The role of parotid gland irradiation in the development of severe hyposalivation (xerostomia) after intensity-modulated radiation therapy for head and neck cancer: Temporal patterns, risk factors, and testing the QUANTEC guidelines. J Craniomaxillofac Surg 2017; 45: 595-600. doi: 10.1016/j. jcms.2017.01.020

24. Eisbruch A, Rhodus N, Rosenthal D, Murphy B, Rasch C, Sonis S, et al. How should we measure and report radiotherapy-induced xerostomia? Semin Radiat Oncol 2003; 13: 226-34. doi: 10.1016/S1053-4296(03)00033-X

25. Jellema AP, Doornaert P, Slotman BJ, Leemans CR, Langendijk JA. Does radiation dose to the salivary glands and oral cavity predict patient-rated xerostomia and sticky saliva in head and neck cancer patients treated with curative radiotherapy? Radiother Oncol 2005; 77: 164-71. doi: 10.1016/j. radonc.2005.10.002

26. Dawes C. Rhythms in salivary flow rate and composition. Int J Chronobiol 1974; 2: 253-79.

27. Wang ZH, Yan C, Zhang ZY, Zhang CP, Hu HS, Tu WY, et al. Impact of salivary gland dosimetry on post-IMRT recovery of saliva output and xerostomia grade for head-and-neck cancer patients treated with or without contralateral submandibular gland sparing: a longitudinal study. Int J Radiat Oncol Biol Phys 2011; 81: 1479-87. doi: 10.1016/j.jjrobp.2010.07.1990

28. Nath R, Anderson LL, Luxton G, Weaver KA, Williamson JF, Meigooni AS Dosimetry of interstitial brachytherapy sources: recommendations of the AAPM Radiation Therapy Committee Task Group No. 43. Med Phys 1995 22: 209-34. doi: $10.1118 / 1.597458$

29. Peppa V, Pappas E, Major T, Takácsi-Nagy Z, Pantelis E, Papagiannis P. On the impact of improved dosimetric accuracy on head and neck high dose rate brachytherapy. Radiother Oncol 2016; 120: 92-7. doi: 10.1016/j. radonc.2016.01.022

30. Nag S, Cano ER, Demanes DJ, Puthawala AA, Vikram B. The American Brachytherapy Society recommendations for high-dose-rate brachytherapy for head-and-neck carcinoma. Int J Radiat Oncol Biol Phys 2001; 50: 1190-8. 